\title{
Phase integrity of zinc oxide doped zirconia under low compacting pressure
}

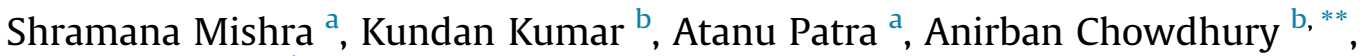 \\ Anushree Roy ${ }^{\text {a, * }}$ \\ a Department of Physics, Indian Institute of Technology Kharagpur, West Bengal, 721302, India \\ ${ }^{\mathrm{b}}$ Metallurgical and Materials Engineering, Indian Institute of Technology, Patna, Bihar, 801106, India
}

\section{A R T I C L E I N F O}

\section{Article history:}

Received 24 March 2020

Accepted 3 June 2020

Available online 11 June 2020

\begin{abstract}
A B S T R A C T
The phase stability of zirconia $\left(\mathrm{ZrO}_{2}\right)$ under external perturbation draws the attention of researchers, as it governs the practical use of this material in technological applications. In this article, the phase transition in zinc oxide $(\mathrm{ZnO})$ doped $\mathrm{ZrO}_{2}$ is addressed by exploiting complementary methodologies, $\mathrm{X}$-ray diffraction, and Raman spectroscopic measurements. We have observed a pressure-induced partial phase transformation in the doped $\mathrm{ZrO}_{2}$ system for a compacting pressure of less than $1 \mathrm{GPa}$. It is noteworthy that the level of compacting pressure used in this study is much lower than the range of pressure, reported in the literature, for observing phase transition in pure and doped $\mathrm{ZrO}_{2}$ using other high-pressure experiments. To understand the underlying dynamics of low pressure-induced phase transformation, we carried out density functional theory calculations for the doped supercell. We have discussed the local bonding environment around the dopant metal ion $\left(\mathrm{Zn}^{2+}\right.$ in the present case), which, we believe, leads to the phase evolution in a real system.
\end{abstract}

() 2020 Elsevier B.V. All rights reserved.

\section{Introduction}

Zirconia $\left(\mathrm{ZrO}_{2}\right)$ is one of the many ceramic materials, which finds a broad range of technological applications [1-4]. The excellent mechanical properties, like, high-temperature strength [3], fracture toughness [2], etc. make it an important candidate for manufacturing various devices, especially in the medical field. These attractive characteristics are, however, largely associated with the tetragonal ( $T$-) and cubic ( $C$-) phases of $\mathrm{ZrO}_{2}$. The monoclinic $(M-)$ phase of $\mathrm{ZrO}_{2}$ is thermodynamically most stable at room temperature and ambient pressure. With an increase in temperature, it undergoes the phase transition by following: $M \rightarrow T \rightarrow C \rightarrow$ melt.

Attempts have been made to stabilize the $T$ - and the $C$ - structure at ambient temperature by appropriate chemical substitution in the $\mathrm{ZrO}_{2}$ matrix. This is accomplished by the replacement of some of the tetravalent $\mathrm{Zr}^{4+}$ ions (ionic radius of $\sim 84 \mathrm{pm}$ [5]) with slightly

\footnotetext{
* Corresponding author.

** Corresponding author.

E-mail addresses: anirban.chowdhury@gmail.com (A. Chowdhury), anushree@ phy.iitkgp.ac.in (A. Roy).
}

larger ions, like, trivalent yttrium ions $\left(\mathrm{Y}^{3+}\right.$ : ionic radius of $\sim 102 \mathrm{pm}$ [5]) or divalent calcium ions $\left(\mathrm{Ca}^{2+}\right.$ : ionic radius of $\left.\sim 100 \mathrm{pm}[5]\right)$ $[6,7]$. In a recent study, the stabilizations of $T$ - and $C$ - phases at room temperature are achieved by doping $\mathrm{Sc}_{2} \mathrm{O}_{3}$ in $\mathrm{ZrO}_{2}$ [8]. Here, the ionic radius of the scandium ion $\left(\mathrm{Sc}^{3+}\right.$ : ionic radius of $\left.\sim 87 \mathrm{pm} \mathrm{[5]}\right)$ is comparable with that of the $\mathrm{Zr}^{4+}$ ion. In the process of aliovalent substitutions, oxygen vacancies are generated to maintain the charge neutrality. The presence of these vacancies leads to internal stress, which, in turn, causes a local distortion in the lattice. The dynamics of phase transformation of doped $\mathrm{ZrO}_{2}$ is complex and strongly depends on external conditions, like the annealing temperature, applied pressure, etc. [6-14].

Reports in the literature on the pressure-induced phase transformations in both undoped and doped $\mathrm{ZrO}_{2}$ systems are quite diverse with a variety of observations and explanations [9,10,15-21]. The most commonly observed pressure-induced phase transition in the case of undoped zirconia is from $M \rightarrow T$ phase and from $T \rightarrow C$-phase [15-20]. Perry et al. [19] had observed a transition from an $M$-phase to a distorted $T$-phase at a pressure of about 4.1 GPa. Also, in the high applied pressure ( $\sim 31 \mathrm{GPa}$ ) regime, an evolution towards a distorted cubic structure has been experimentally observed [15]. Density functional theory (DFT) studies on the undoped system revealed that the pressure-induced phase 
transition proceeds from an $M$-phase to a $T$-phase via a structurally unstable orthorhombic phase [20]. Further calculations show that for the $T$-phase, externally applied pressure removes tetragonal distortions, and the structure transforms into a $C$ - phase [17].

For doped $\mathrm{ZrO}_{2}$, however, the mechanism of phase transformation is very different from that of the undoped compound. Though in the literature, a large number of articles discussed the phenomenon using a variety of dopants like $\mathrm{Y}_{2} \mathrm{O}_{3}, \mathrm{CaO}, \mathrm{Sc}_{2} \mathrm{O}_{3}$, etc. [6-10], the contradicting reports hinder us to conclusively comment on the origin of the phase transition in this system. Phase transformation in the doped system not only depends on the dopant type but also on the relative concentration of the dopant. While the phase transition could not be observed in phase stabilized $\mathrm{Y}_{2} \mathrm{O}_{3}\left(5.3 \mathrm{wt} \%\right.$ ) doped $\mathrm{ZrO}_{2}$ under different compressive stress (0-0.9 GPa) [10], the low pressure-induced (0.1 GPa) martensitic phase transformation from the metastable $T \rightarrow M$-phase has been reported by Majumder and Chatterjee [21] in compacted $\mathrm{Y}_{2} \mathrm{O}_{3}$ ( $3 \mathrm{~mol} \%$ ) doped $\mathrm{ZrO}_{2}$. In this case, the phase transformation is mediated by the segregation of the dopants to surface and grain boundaries. On the other hand, for 3 wt $\% \mathrm{Y}_{2} \mathrm{O}_{3}-\mathrm{ZrO}_{2}$ system [a phase mixture of $M-(\sim 80 \%)$ and $\left.T-(\sim 20 \%) \mathrm{ZrO}_{2}\right], M \rightarrow T-\mathrm{ZrO}_{2}$ transformation was observed on the application of pressure of 4.2 GPa; the final T-phase content was noted to be $~ 85 \%$ [9].

In this article, we probe the possibility of utilizing a multifunctional oxide, like $\mathrm{ZnO}$ for stabilizing the $\mathrm{ZrO}_{2}$ matrix to a desired structural phase. As $\mathrm{Zn}^{+2}$ is a divalent ion, more oxygen vacancies (1 vacancy per dopant) are expected to be bound within the local structure of the doped compound than what we find in the cases of $\mathrm{Y}_{2} \mathrm{O}_{3}$ and $\mathrm{Sc}_{2} \mathrm{O}_{3}$ doping (1 vacancy per 2 dopants). It is also nontrivial to compare the process of phase transition as a result of doping of two different divalent ions. The ionic radius of $\mathrm{Zn}^{2+}(60$ $\mathrm{pm}[5])$ is much less than that of other divalent ions like $\mathrm{Ca}^{2+}$. Hence, the lattice distortion, for compensating the volume reduction due to oxygen vacancies, is expected to be different in the case of $\mathrm{CaO}$ or $\mathrm{ZnO}$ doping, which can affect the phase stabilization of the system. For proper utilization of these materials in devices (in the form of bars, rods, etc.), the fabrication procedures require compacting pressure on the powder samples. Thus, it is important to investigate the underlying physics governing the phase stability of doped $\mathrm{ZrO}_{2}$ under compaction for the efficient utilization of the material.

We have investigated the effect of compacting pressure on the phase transformation of the $\mathrm{ZnO}$ doped $\mathrm{ZrO}_{2}$ of a mixed $C / T$ phase, using X-ray diffraction (XRD) and Raman spectroscopic measurements. Our experimental observations suggest the formation of pressure-induced $M$-phase and distorted $T$-phase in the host matrix. Raman spectroscopy is a powerful tool to detect the changes in the atomic bonding of a system under external perturbation $[6,8-11,15,19]$. It is particularly sensitive to the polarizability of the oxygen ions. As the oxygen is expected to play a crucial role in phase transformations in $\mathrm{ZrO}_{2}$, Raman spectroscopy can serve as a useful tool for studying the stability of the compound. In our study, while XRD measurements probe the overall structural phase in the system, Raman scattering yields the information of local structural disorder. Thus, these two complementary techniques render integral information of the structural stability of the compound. Furthermore, we have carried out DFT calculations to investigate the chemical bonding mechanism in $\mathrm{ZnO}$ doped $\mathrm{ZrO}_{2}$, and the possible underlying dynamics of the experimentally observed phase transformation. There are few articles in the literature in which the formation and applications of $T$-phase stabilized by $\mathrm{ZnO}$ doped $\mathrm{ZrO}_{2}$ are reported [11-13]. To the best of our knowledge, the effect of external compaction pressure on this doped compound is still missing in the literature. Noteworthy is the fact that the phase transformation, which we will discuss in this article, is irreversible in nature and has been observed at low pressure $(<1 \mathrm{GPa})$. The specific role of mechanical stress to a phase transition during pelletization (compaction) may be different from the same reported using other high-pressure laboratory-based experiments (say, in the case of hydrostatic pressure-induced phase transition in doped and undoped $\mathrm{ZrO}_{2}$ using a diamond anvil cell $\left.[9,10,15,17]\right)$. However, it is to be noted that the range of pressure used in this study is much lower than the pressure used in the literature to investigate the phase stability of various doped $\mathrm{ZrO}_{2}$ compounds. The applied stress is very close to the nominal mechanical stress generally applied during normal processing operations (e.g., shaping, pressing of a green body). Our results indicate that the phase stability issues of the present system can influence these normal processing operations.

\section{Experimental and simulation methodologies}

$8 \mathrm{~mol} . \% \mathrm{ZnO}$ doped $\mathrm{ZrO}_{2}$ powder sample was synthesized using a co-precipitation method. The dried precipitates were calcined at $500{ }^{\circ} \mathrm{C}$ in the air for $2 \mathrm{~h}$. We refer to this as-prepared powder as Sample A. For pressure dependent studies, the powder samples were pelletized at room temperature by pressing the powder between steel dies of diameter $10 \mathrm{~mm}$. The applied pressure was in the range of $0.05 \mathrm{GPa}-0.95 \mathrm{GPa}$. We refer to the compacted samples, under $0.05,0.15,0.25,0.35,0.45,0.55,0.65,0.75,0.85$ and 0.95 GPa applied pressures, as Sample B-Sample K, respectively. The detail of the sample preparation procedure is available elsewhere [22].

The XRD measurements were carried out in $\theta-2 \theta$ geometry using $\mathrm{Cu} \mathrm{K} \alpha$ radiation ( $\lambda=1.5406 \AA$, Rigaku TTRX-III, Japan). The diffraction angle $(2 \theta)$ was selected from $20^{\circ}$ to $80^{\circ}$ with a step size of $0.02^{\circ}$ and a scan rate of $0.2^{\circ} / \mathrm{min}$. The refinement of the XRD data was carried out using FULLPROF [23], a free software available online. The background was obtained using linear interpolation between 50 selected points. A Cauchy (Lorentzian) function was used to describe the peak shape. Various parameters, like the profile parameters, the scale factor, and the lattice parameters of different phases, were refined. For the refinement, the initial structural starting parameters for different phases were chosen from the corresponding JCPDS files.

Micro-Raman spectra were recorded at room temperature using a $532 \mathrm{~nm}$ excitation wavelength of a diode laser. The spectrometer was equipped with an optical microscope (model BX41, Make Olympus, Japan), a single monochromator (model IHR550, Make Horiba, France), and a Peltier cooled CCD $(1024 \times 256$ pixels, model Synapse, Make Horiba, France) detector. For wavelengthdependent measurements, Raman spectra were recorded using several excitation wavelengths between $457.9 \mathrm{~nm}$ and $647.1 \mathrm{~nm}$ from $\mathrm{Ar}^{+}-\mathrm{Kr}^{+}$laser (model Innova 70C, Coherent, USA). The measurements were carried out in back-scattering geometry using a triple monochromator (model T64000, Make JY, Horiba, France). In addition, Raman spectra of Sample K were recorded using $632 \mathrm{~nm}$ and $785 \mathrm{~nm}$ wavelengths of diode lasers and a single monochromator spectrometer (HR-800, Make JY, Horiba, France).

Furthermore, we carried out the first-principles calculations to estimate the $\mathrm{Zr}-\mathrm{O}$ bond lengths in undoped and $\mathrm{ZnO}$ doped $\mathrm{ZrO}_{2}$ systems using all-electron-full potential Wien2K code [24]. The simulations are carried out in the athermal limit, i.e, $T=0 \mathrm{~K}$. For the optimization of the structures, we considered generalized gradient approximation (GGA) functional as mentioned in Ref. [25]. The spherical harmonic function inside the muffin-tin spheres was limited by $l_{\max }=12$, where the muffin-tin radii for $\mathrm{Zr}$, $\mathrm{O}$ and $\mathrm{Zn}$ were fixed at 2.0, 1.6, and 1.95 a.u (atomic units), respectively. In interstitial regions, the charge density and potential were defined through $G_{\max }$ at $12 \mathrm{Bohr}^{-1}$. The $R_{\min }^{M T} K_{\max }$ was fixed to 8 for all 
calculations. The $k$-mesh for the calculation was $4 \times 4 \times 3$. The force on each atom was relaxed by less than $0.5 \mathrm{mRyd} / \mathrm{a}$.u.

\section{Results}

\subsection{X-ray diffraction}

The XRD patterns of as-prepared powder of $\mathrm{ZnO}$ doped $\mathrm{ZrO}_{2}$ (Sample A) and samples compacted at different pressures (Sample B-Sample K) are shown in Fig. 1. All XRD patterns were analyzed using Rietveld refinement. The refined XRD patterns for few characteristic samples are shown in Fig. 2 [Sample A (as-prepared powder), Sample G (compacted at $0.55 \mathrm{GPa}$ ), and Sample K (compacted at $0.95 \mathrm{GPa})]$.

The XRD patterns of $C$-phase (JCPDS file no.49-1642) and $T$ phase (JCPDS file no. 50-1089) of $\mathrm{ZrO}_{2}$ are very similar. The earlier report in the literature [22] suggests that in $\mathrm{ZnO}$ doped $\mathrm{ZrO}_{2}$ both these phases are equiproportional. In the present analysis too, the best refinement of the data points could be obtained by taking into account a mixed contribution of $C$ - and $T$-phase (with space group $F m \overline{3} m$, and $P 4_{2} / n m c$, respectively) (see Fig. 2(a) and Fig. S1 in the Supplementary Section for details). Similarly, the XRD patterns for Sample B and Sample $C$ were best fitted with a mixed contribution of $C$ - and $T$-phases. In Fig. 2, $C$ - and $T-\mathrm{ZrO}_{2}$ phases are indexed by green and orange bars, respectively. From the refinement of the pattern of Sample A, the lattice constants for the $T$-phase are found to be $a_{T}=3.610 \AA$ and $c_{T}=5.171 \AA$. For the $C$-phase, the lattice constant $a_{C}$ is estimated to be $5.094 \AA$. These values compare well

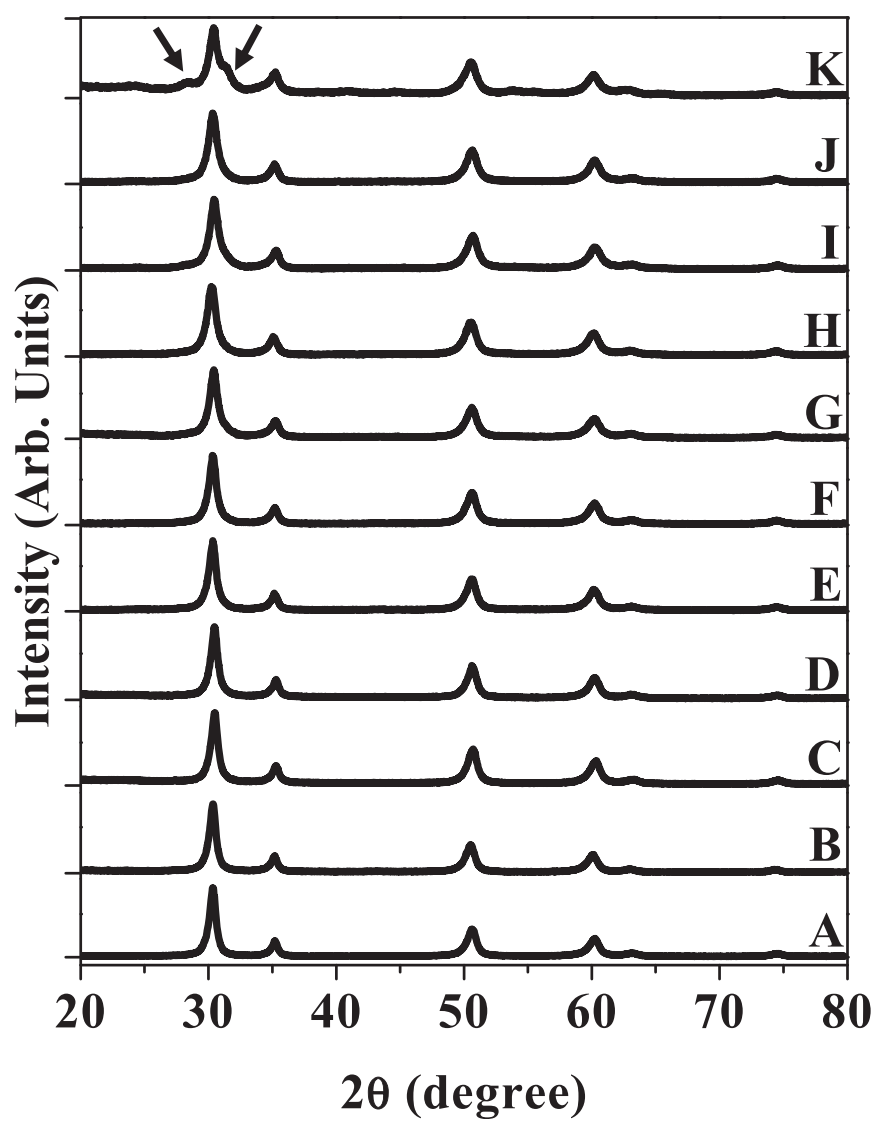

Fig. 1. XRD patterns of the as-prepared powder sample (Sample A) and the pellets made by compacting the sample at various pressures in the range $0.05-0.95 \mathrm{GPa}$ (Sample B-Sample K). The additional features, which clearly appear in the pattern for the Sample K are shown by arrows in the topmost panel. with those published in the open literature [26,27]. For the Sample $\mathrm{K}$ (i.e., the sample compressed at the highest pressure), new features in the pattern could be clearly observed at both low and high diffraction angles (at $\sim 28.4^{\circ}$ and $\sim 31.4^{\circ}$, respectively) with respect to the main peak (see the arrows for Sample K in Figs. 1 and 2). Hence, it was necessary to include the contribution of an additional phase (along with $T$ - and $C$ - phase) for the refinement. For $M-\mathrm{ZrO}_{2}$, the (111) and (111) diffraction peaks appear at $\sim 28.2^{\circ}$ and $\sim 31.4^{\circ}$, respectively (with reference to the JCPDS file no. 37-1484). First, the pattern was fitted with $T$-, $C$ - and $M$-phase, and the value of $\mathrm{R}_{\mathrm{wp}}$ (weight profile $\mathrm{R}$ value) for the best fit was $15.7 \%$ (with $\chi^{2}=2.97$ ). The difference pattern showed a significant residual count at $\sim 31.4^{\circ}$. For nanocrystalline undoped $T-\mathrm{ZrO}_{2}$ pelletized at a pressure of 1.0 and $3.4 \mathrm{GPa}$, a peak appears at $31.4^{\circ}$, which has been attributed in the literature as the $T^{\prime}$ - phase (indexed with the JCPDS 13-4748) [17]. It can be considered as a new tetragonal modification of the $T$ phase, induced purely by the applied pressure. Thus, the diffraction pattern of the Sample K was refined with four phases, $T-, C-, M$ - and $T^{\prime}$ - phase and the value of $R_{\mathrm{wp}}$ for the best fit was reduced to $14 \%$ (with $\chi^{2}=2.65$ ) (Refer to Fig. S2 of the Supplementary Section). In Fig. 2, the Bragg peaks of $M$ - and $T^{\prime}$ - phase are indexed by purple and cyan bars, respectively. Similarly, the XRD patterns of Sample $\mathrm{D}$-Sample $\mathrm{J}$ could be better refined by including the above four phases in our analysis. The refined pattern of the Sample $G$ is also shown in Fig. 2. Table 1 lists the $T$-, $C$-, $M$ - and $T^{\prime}$ - phase fractions in all samples under study, as obtained from the Rietveld refinement of the XRD data. From Table 1, it can be observed that up to a compacting pressure of $0.85 \mathrm{GPa}$ (Sample J), the contributions of both $M$ - and $T^{\prime}$ - phases in the diffraction patterns are either nil or significantly less than the same of $T$ - and $C$ - phases. In fact, for Sample D-Sample H, the minor phase (i.e., $M$ - and $T^{\prime}$-phases) fractions are negligible $(\sim 1 \%)$. The significant presence of $M$-phase in all samples as observed in our Raman measurements (shown later) and the possibility of the existence of $T^{\prime}$-phase in the doped structure as obtained from DFT calculations (discussed later) compelled us to consider the contributions of these minor phases in the refinement of the XRD data, unless these additional phases increased the $\chi^{2}$ values of the fitting procedure or yielded unphysical values of the refined parameters.

Fig. 3(a) plots the evolution of $T-, C-, M$ - and $T^{\prime}$ - phase fractions with compacting pressure. The corresponding symbols for $C_{-}, T-, M-$ and $T^{\prime}$ - phase are squares $(\square)$, down triangles $(\nabla)$, up triangles $(\boldsymbol{\Delta})$, and circles ( () , respectively. We find that new phases ( $T^{\prime}$ - and $M-$ ) evolve in the system at the expense of the tetragonal phase, whereas the cubic phase fraction remains nearly unchanged. This is not surprising, as the high symmetry $C$-phase is more stable than a $T$-structure [18]. Fig. 3(b) plots the change is the lattice constants of $T$ - and $C$ - phases in the system with the increase in the compacting pressure. The open symbols show the lattice constant $a_{C}$ of the $C$ phase, whereas, the filled symbols present the same $\left(a_{T}\right.$ and $\left.c_{T}\right)$ for the $T$-phase. It can be seen that under compacting pressure, the lattice parameter $\left(a_{C}\right)$ of the $C$-phase remains nearly unchanged (osymbols in Fig. 3(b)). The lattice parameter $c_{T}$ of the $T$-phase decreases beyond a certain compacting pressure ( symbols in Fig. 3(b)), while the value of $a_{T}$ remains nearly constant ( $\square$ symbols in Fig. 3(b)).

It is noteworthy that the effect is observed on the green body, i.e., even without sintering the ceramic. In other words, the effect of temperature, which is commonly associated with such phase transitions, has no relevance in the present case; only the applied external pressure ascertains the observed phase transition. Secondly, the applied mechanical pressure is an order of magnitude smaller than the same reported in the literature for the phase transition in the related systems. 


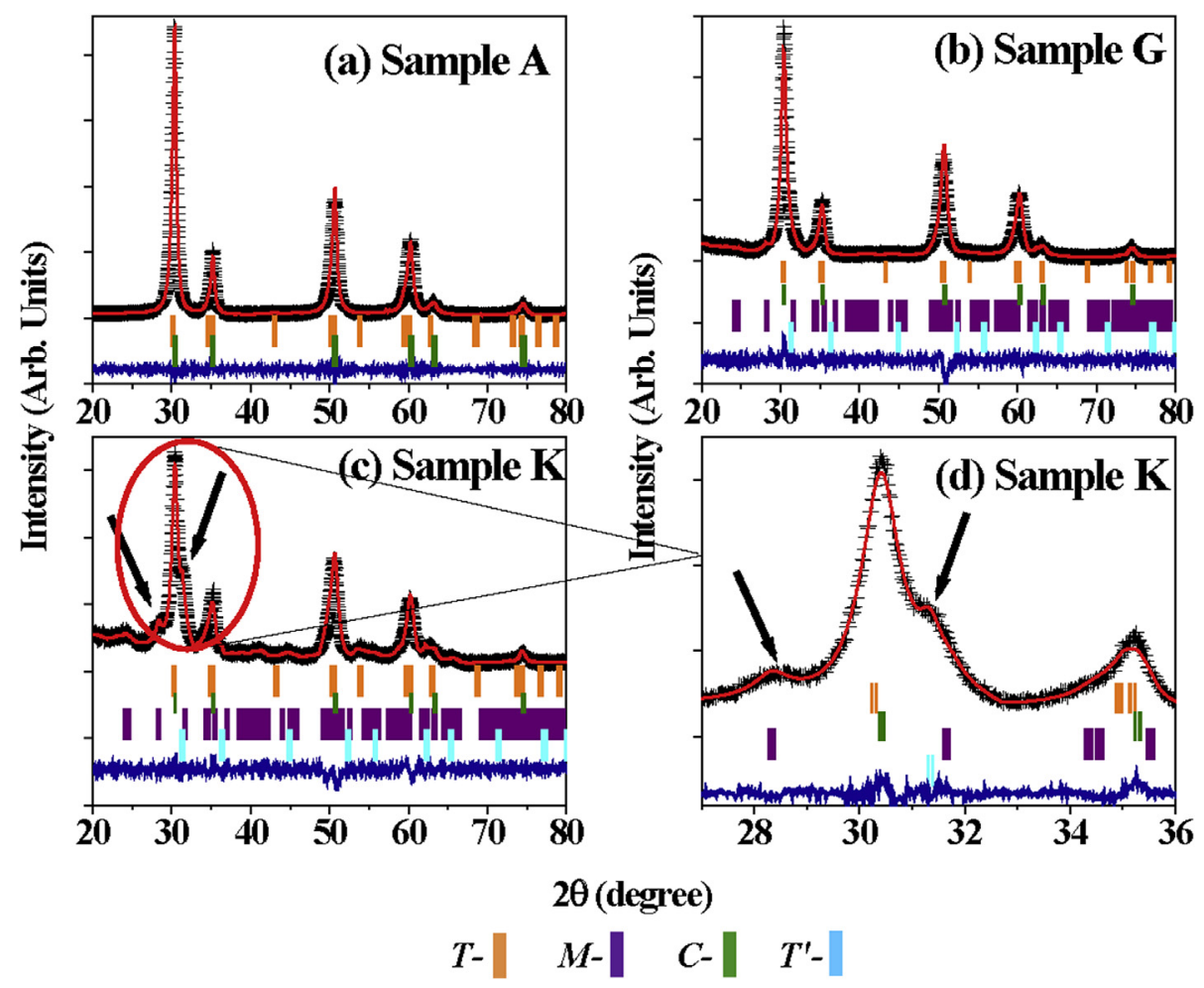

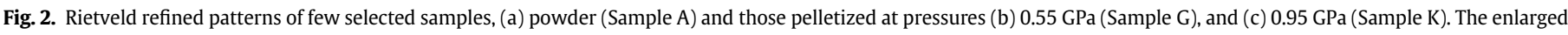

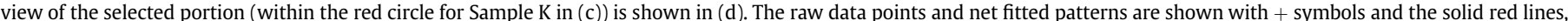

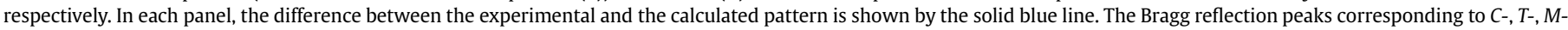

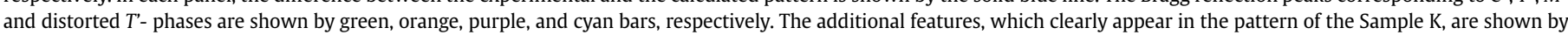

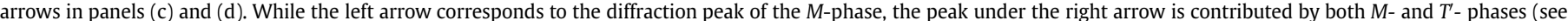
the text). (For interpretation of the references to colour in the figure legend, the reader is referred to the Web version of this article)

\subsection{Raman scattering}

To obtain further insight into the evolution of new phases in $\mathrm{ZnO}$ doped $\mathrm{ZrO}_{2}$ under compacting pressure, as observed from XRD measurements, room temperature Raman spectra of the asprepared powder sample (Sample A) and samples compacted under different mechanical pressures (Sample B to Sample K) were measured using $532 \mathrm{~nm}$ excitation wavelength and are shown in Fig. 4. For clarity, the whole spectral range is divided into two regions. We define the range from $125 \mathrm{~cm}^{-1}$ to $400 \mathrm{~cm}^{-1}$ as Region I and from $400 \mathrm{~cm}^{-1}$ to $750 \mathrm{~cm}^{-1}$ as Region II. In the spectral range between 125 and $750 \mathrm{~cm}^{-1}$, several zone center $(\Gamma)$ phonon modes of different phases of $\mathrm{ZrO}_{2}$ contribute to the Raman spectra $[9,10]$. The frequencies of these vibrational modes of different phases of $\mathrm{ZrO}_{2}$ are available in Table 2. Each spectrum in Fig. 4 is deconvoluted by considering a Lorentzian function for each expected mode and net Raman spectrum in each panel was fitted with $I(\omega)=$ $\sum_{j=1}^{N} A_{j} \frac{\Gamma_{j}}{\left(\omega-\omega_{j}\right)^{2}+\Gamma_{j}^{2}}$, where $A_{j}, \Gamma_{j}, \omega_{j}$ are integral intensity, width, and Raman peak position for the $j$ th observed mode, $I(\omega)$ is the net intensity, and $N$ is the total number of modes. The Region-I and II are deconvoluted with eight $(N=8)$ and seven $(N=7)$ Lorentzian functions, respectively. In Fig. 4, solid orange lines are the deconvoluted components for the $T$-phase, and the solid purple lines are the same for the $M$-phase. The net fitted spectra are shown by the solid green lines. Since a large number of Raman peaks appear in the spectra and many of them nearly overlap, during the fitting procedure, we allowed the peak positions to vary only by $5 \%$ from the listed expected values in Table 2. Interestingly, though from XRD measurements the $M$-phase could be clearly detected only for the samples compacted at high pressure, the best fit of the Raman spectrum even of Sample A (as-prepared powder) could be obtained only after including the contribution of the Raman modes of the $M$-phase along with the modes of the $T$-phase and $C$-phase. This is not surprising as Raman spectroscopy is more sensitive than XRD measurements in detecting the $M$-phase of zirconia [28]. For example, for yttria-stabilized zirconia blocks, at a given composition, the $M$-fraction obtained by Raman spectroscopy is $\sim 1.5-2$ times higher than the same obtained from XRD measurements [29]. Referring to Table 2, we find that over the spectral range, shown in Fig. 4, the $C$ - phase of $\mathrm{ZrO}_{2}$ exhibits only one $\mathrm{F}_{2 \mathrm{~g}}$ Raman mode at $\sim 610 \mathrm{~cm}^{-1}$ [10]. It is also to be noted that very close to this Raman mode of the $C$-phase, $A_{1 g}$ mode of the $T$-phase and $B_{g}$ mode of the $M$-phase appear at $613 \mathrm{~cm}^{-1}$ and $\sim 615 \mathrm{~cm}^{-1}$, respectively (refer to Table 2). Because of the large spectral width of the individual mode, these Raman peaks are expected to overlap in the recorded spectra. Thus, the deconvoluted component at $615 \mathrm{~cm}^{-1}$ (shown by the blue line) can be contributed either by $C$ - or $M$ - or $T$-phase or by all. Hence, from Raman spectral analysis, it is non-trivial to decipher the contribution of the $C$-phase, as revealed from the XRD measurements. Similarly, the Raman peak at $635 \mathrm{~cm}^{-1}$ of the $M$-phase is very close to the Raman mode of the $T$-phase at $640 \mathrm{~cm}^{-1}$ (refer to Table 2). Therefore, we refrain from assigning the Raman peak at $640 \mathrm{~cm}^{-1}$ (shown by the black line) to a specific phase. For a better visual clarity on the evolution of the $T$ - and $M$-phases with the applied pressure, the deconvoluted components in the spectra for Sample A (bottom-most spectrum) and Sample K (just below the topmost spectrum) are shown by orange filled and purple filled regions for $T$ - and $M$-phases, respectively. The mode, for which all three phases ( $T$-, $M-$, and $C_{-}$) may contribute, is shown by the cyan 

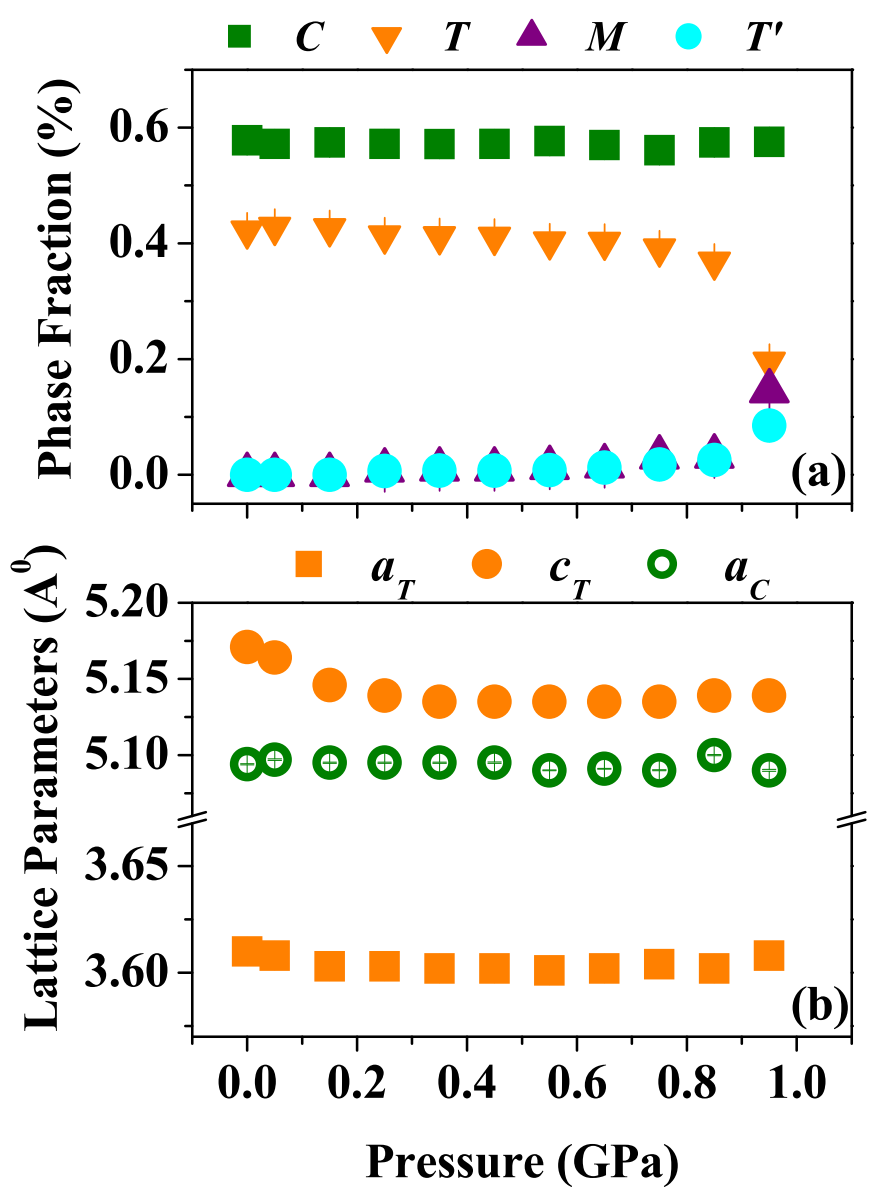

Fig. 3. (a) Evolution of $T-, C_{-}, M-$, and $T^{\prime}$ - phase fractions with compacting pressure. The corresponding symbols for $C-, T-, M-$, and $T^{\prime}-$ phase are solid square ( $\square$ ), solid down triangle $(\nabla)$, solid up triangle $(\boldsymbol{\Delta})$ and solid circle $(\odot)$, respectively. (b) The variation is the lattice constants of $T$ - and $C$ - phases in the system with compacting pressure. The lattice constant $a_{C}$ for $C$-phase is given by open symbol (o), whereas, lattice constants $a_{T}$ and $c_{T}$ of the $T$-phase are given by solid symbols, and $\odot$, respectively. The error bars to the data points (standard deviation of the fitted parameters) are nearly within the size of the symbols. (For interpretation of the references to colour in this figure legend, the reader is referred to the Web version of this article.)

filled area and the mode for which both $M$ - and $T$-phases are expected to contribute is shown by the grey filled area. Here we would like to mention that the pressure-induced modified $T$-phase, i.e. $T^{\prime}$ - phase, as observed from XRD measurements in Fig. 2, is expected to give rise to new modes (which are Raman inactive for the $T$-phase) due to the breakdown of the selection rules or a change in the spectral line profile. The broad spectral line shapes

restricted us to detect these changes in the net spectral profile. Thus, the presence of the $T^{\prime}$ - phase in the compacted samples could not be revealed from their Raman spectra.

Though some of the structural information, for example, the formation of $T^{\prime}$-phase, $T: C$ phase ratio, obtained from XRD measurements could not be revealed by the Raman data, we could exploit the later technique to find some other complementary information of the doped system, which could not be observed from the diffraction patterns. A careful look into the deconvoluted components in Fig. 4 indicates an increase in the $M$-phase with an increase in the mechanical pressure on the sample (i.e., from Sample B to Sample K). To study the $M$ - and $T$-fraction in each sample and their evolution with the applied pressure, we have estimated the ratio of the integral intensity, as obtained from the curve fitting program, of each mode $\left(I_{i}, i=M\right.$ or $T$ for the modes related to $M$-phase or $T$-phase) with respect to the total intensity $\left(I_{\text {tot }}\right)$ of all modes. The changes in these ratios for some prominent $M$ - and $T$-modes with applied pressure are shown in Fig. 5. The error bar to each data point is estimated by using the standard deviation of the intensities, as a fitting parameter, and then using the propagation of error methodology. As the Raman modes are broad and overlapped, the fitting parameters are not unique. Hence, there is a large scattering of the data points in Fig. 5. Nonetheless, we observe a trend for the intensity ratio $I_{M} / I_{\text {tot }}$ and $I_{T} /$ $I_{\text {tot }}$. The $M$-phase fraction gradually increases with pressure (Fig. 5(a)-(f)) with a concurrent reduction in the $T$-phase fraction (Fig. 5(g)-(i)).

To investigate whether the $M$-phase has formed only near the surface or even in bulk, we have carried out wavelength dependent Raman measurements for the sample compacted at the highest pressure (Sample K (compacted at highest pressure $0.95 \mathrm{GPa}$ )). The variation of both $T$-fraction $\left[\left(I_{148} / I_{\text {tot }}\right)\right.$, obtained for the $T$-mode at $\sim 148 \mathrm{~cm}^{-1}$ ] and $M$-fraction [( $\left.I_{175} / I_{\text {tot }}\right)$ for the $M$-mode at $\sim 175 \mathrm{~cm}^{-1}$ ] with excitation wavelengths are shown in Fig. 6(a) and (b), respectively. The error bars to the data points are obtained by following the same procedure as in Fig. 5 . It is to be noted that the Raman peaks at 175 and $148 \mathrm{~cm}^{-1}$ are two distinct Raman features for the $M$ - and $T$-phases in the sample respectively, and least contributed by the other Raman modes.

Wavelength dependent intensity of the Raman spectrum depends on various factors, like the penetration depth of the beam and the absorption coefficient of the material. It also varies inversely with the fourth power of laser wavelength. As we normalized the intensity of the above mentioned Raman modes with $I_{\text {tot }}$, we expect the ratio to remain constant for all wavelengths in case of the same phase fraction in the probing depth. We find in Fig. $6(\mathrm{a})$ that the intensity ratio $I_{148} / I_{\text {tot }}$, which indicates the $T$-phase fraction in the sample, increases beyond $647 \mathrm{~nm}$ as the excitation wavelength. However, for the ratio $I_{175} / I_{\text {tot }}$, which carries the

Table 1

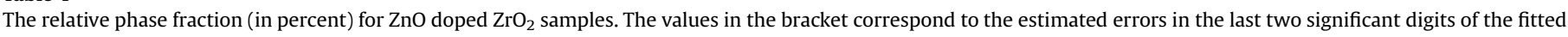
values obtained from Rietveld refinement.

\begin{tabular}{|c|c|c|c|c|c|}
\hline Sample & Applied pressure (GPa) & Tetragonal $(T)$ phase & Cubic $(C)$ phase & Monoclinic $(M)$ phase & Distorted tetragonal $\left(T^{\prime}\right)$ phase \\
\hline A & 0 (powder) & $42.20(27)$ & $57.80(31)$ & - & - \\
\hline B & 0.05 & $42.82(35)$ & $57.18(50)$ & - & - \\
\hline C & 0.15 & $42.57(42)$ & $57.43(66)$ & - & - \\
\hline $\mathrm{D}$ & 0.25 & $41.35(28)$ & $57.19(57)$ & $0.81(20)$ & $0.65(12)$ \\
\hline $\mathrm{E}$ & 0.35 & $41.20(35)$ & $57.14(68)$ & $0.95(12)$ & $0.71(12)$ \\
\hline $\mathrm{F}$ & 0.45 & $41.11(38)$ & $57.19(72)$ & $0.98(20)$ & $0.73(12)$ \\
\hline G & 0.55 & $40.33(43)$ & $57.65(25)$ & $1.23(12)$ & $0.80(18)$ \\
\hline $\mathrm{H}$ & 0.65 & $40.26(83)$ & $56.98(88)$ & $1.50(12)$ & $1.25(22)$ \\
\hline I & 0.75 & $39.07(82)$ & $56.08(58)$ & $3.07(22)$ & $1.78(16)$ \\
\hline $\mathrm{J}$ & 0.85 & $36.83(25)$ & $57.42(69)$ & $3.20(38)$ & $2.54(13)$ \\
\hline K & 0.95 & $19.50(25)$ & $57.52(30)$ & $14.47(40)$ & $8.50(26)$ \\
\hline
\end{tabular}




\section{Region: I}

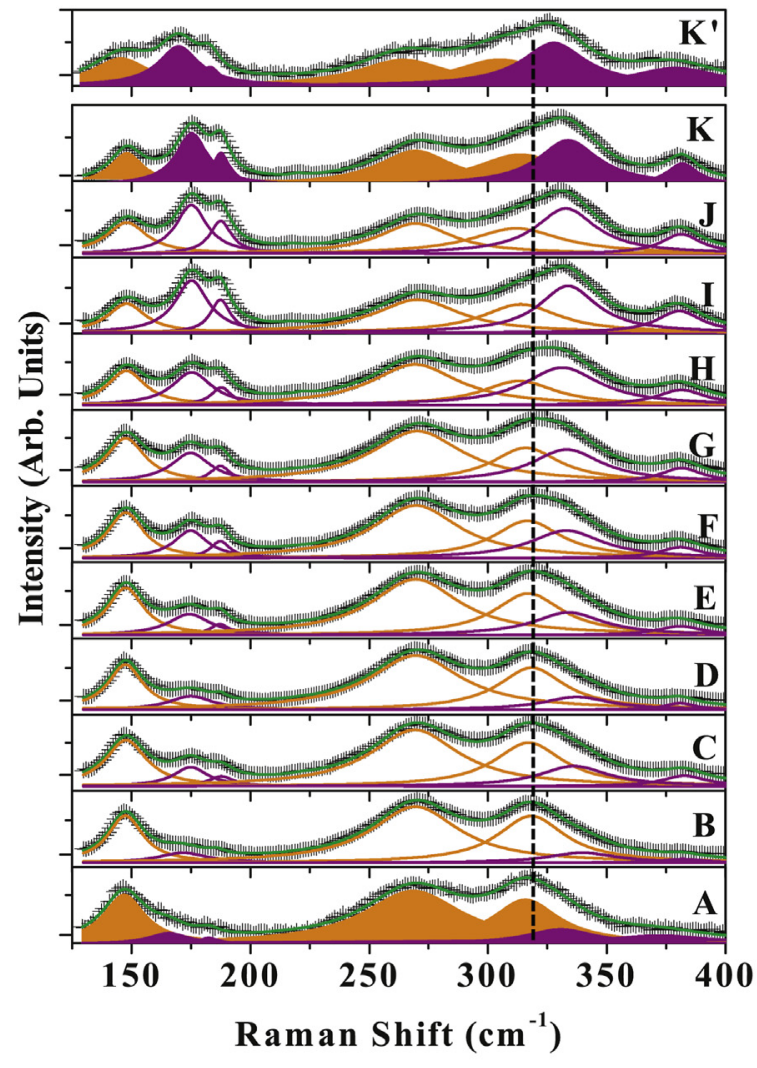

\section{Region: II}

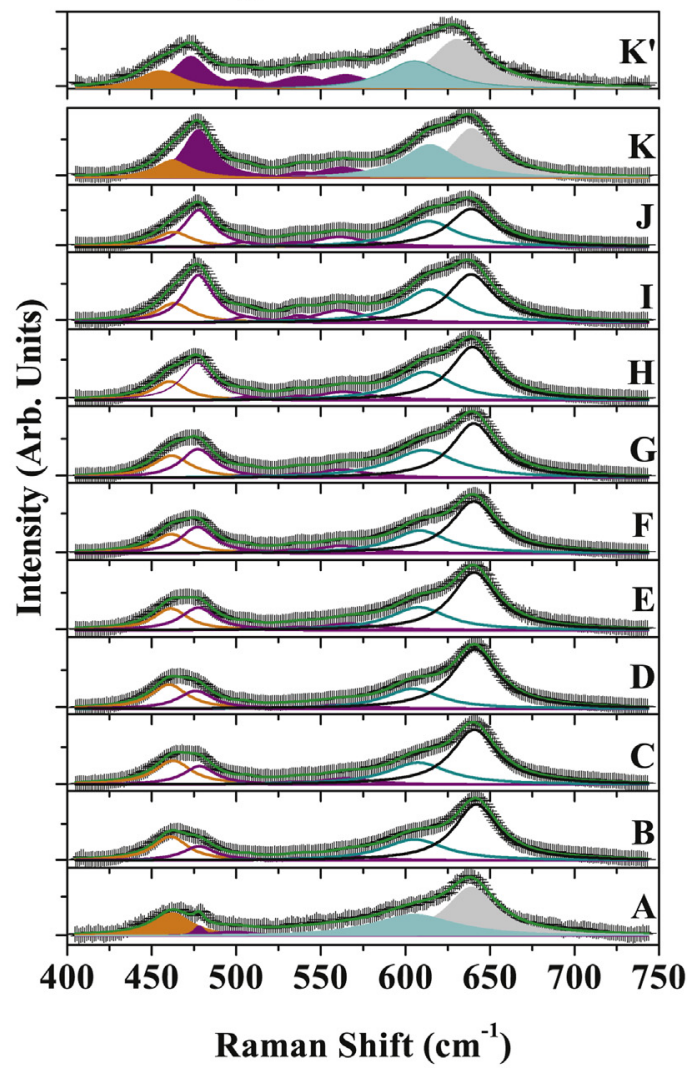

$\boldsymbol{M}+\boldsymbol{T}+\boldsymbol{C}-$

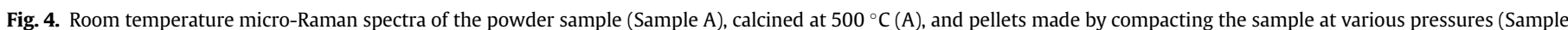

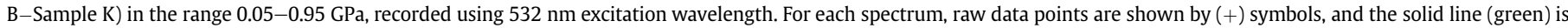

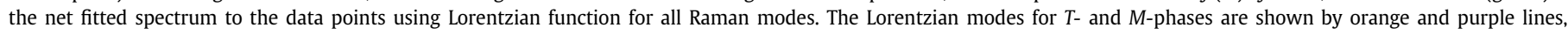

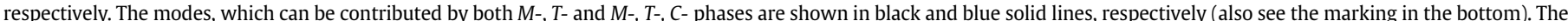

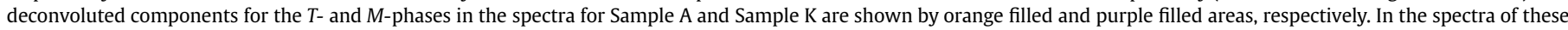

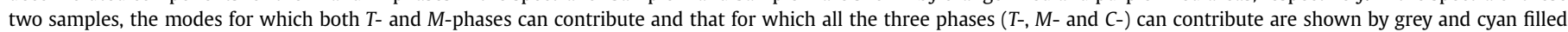

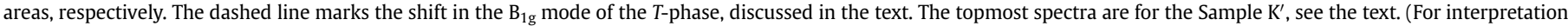
of the references to colour in this figure legend, the reader is referred to the Web version of this article.)

signature of the $M$-phase fraction, we observe a significant drop in the intensity ratio beyond the above excitation wavelength $(647 \mathrm{~nm})$. The penetration depth of light of wavelength $632 \mathrm{~nm}$ in $\mathrm{ZrO}_{2}$ is $\sim 34 \mu \mathrm{m} \mathrm{[30].} \mathrm{Though} \mathrm{the} \mathrm{number} \mathrm{of} \mathrm{data} \mathrm{points} \mathrm{above}$ $650 \mathrm{~nm}$ is low due to unavailability of single-mode laser excitation wavelengths required for Raman measurements, Fig. 6 strongly indicates that the observed phase transformation (as revealed from XRD and Raman scattering measurements) dominates over this depth in the surface-subsurface regime of the compacted blocks.

The $\mathrm{B}_{1 \mathrm{~g}}$ mode at $\sim 322 \mathrm{~cm}^{-1}$ for the $T-\mathrm{ZrO}_{2}$ (Refer to Table 2 and the dashed line in Fig. 4) involves sublattice expansion of zirconia [17]. Under harmonic approximation in an ionic crystal, polyhedral compressibility ( $\kappa)$ can be written as [17].

$\kappa=0.46 \frac{\Delta \nu}{\nu} \frac{1}{\Delta P}$

Here, $v$ is the Raman shift of the $\mathrm{B}_{1 \mathrm{~g}}$ mode, and $\Delta v$ corresponds to the change in Raman shift due to the change in the pressure $\Delta P$. In Fig. 7 we plot the variation in Raman shift of the $B_{1 g}$ mode (as obtained from the curve fitting) with the applied pressure (i.e., for

Table 2

Assignment of Raman modes of $M-, C$ - and $T-\mathrm{ZrO}_{2}[9,10]$.

\begin{tabular}{|c|c|c|c|c|c|c|c|c|c|c|}
\hline \multirow[t]{4}{*}{ Phase } & \multicolumn{10}{|c|}{ Frequency $\left(\mathrm{cm}^{-1}\right)$ and Mode Assignment } \\
\hline & \multicolumn{5}{|c|}{ Pure Modes } & \multicolumn{5}{|c|}{ Mixed Modes } \\
\hline & \multicolumn{2}{|c|}{$\begin{array}{c}\text { Colour code orange in } \\
\text { Fig. } 4\end{array}$} & \multicolumn{3}{|c|}{ Colour code purple in Fig. 4} & \multicolumn{3}{|c|}{ Colour code cyan in Fig. 4} & \multicolumn{2}{|c|}{$\begin{array}{l}\text { Colour code } \\
\text { grey in Fig. } 4\end{array}$} \\
\hline & $\mathbf{B}_{1 \mathrm{~g}}$ & $\mathbf{E}_{\mathrm{g}}$ & $A_{g}+B_{g}$ & $\mathbf{A}_{\mathrm{g}}$ & $\mathbf{B}_{\mathrm{g}}$ & $\mathbf{F}_{2 g}$ & $A_{1 g}$ & $\overline{\mathbf{B}_{\mathrm{g}}}$ & $\mathbf{E}_{\mathbf{g}}$ & $\mathbf{A}_{\mathrm{g}}$ \\
\hline Cubic & - & - & - & - & - & 610 & - & - & - & - \\
\hline Tetragonal & 148322 & 261464 & - & - & - & - & 613 & - & 640 & - \\
\hline Monoclinic & - & - & 179381 & 190304346474557 & 220333500536 & - & - & 615 & - & 635 \\
\hline
\end{tabular}




\section{Monoclinic Modes}
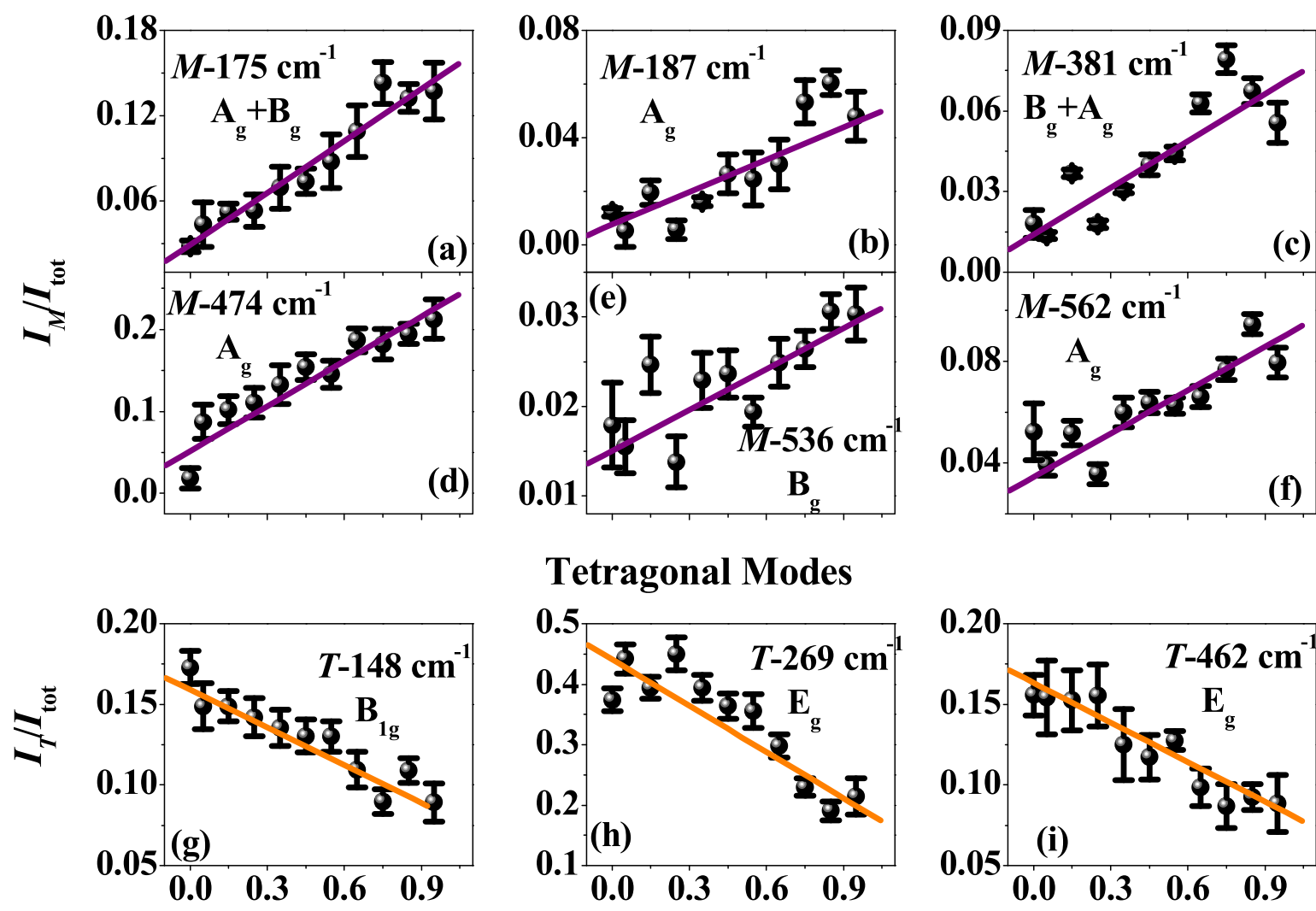

\section{Tetragonal Modes}

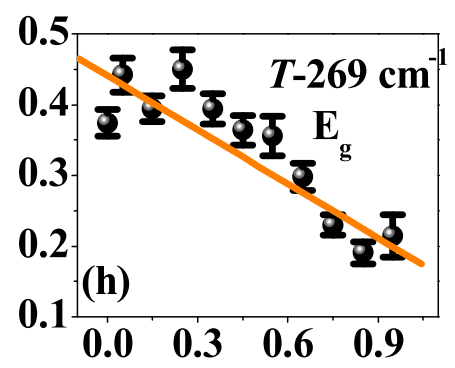

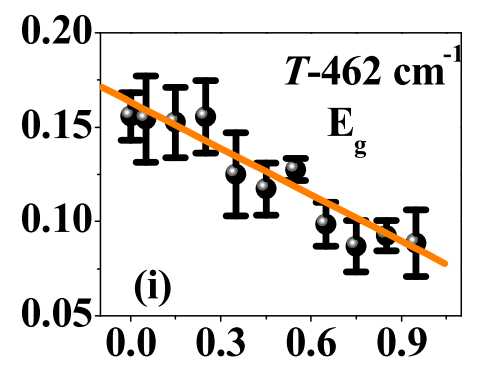

\section{Pressure (GPa)}

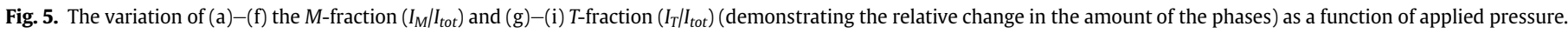

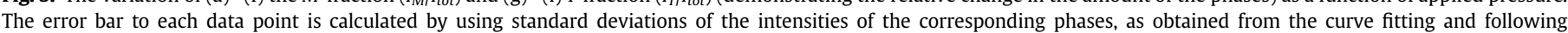

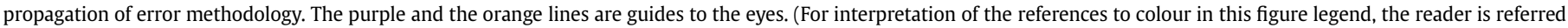
to the Web version of this article.)

Sample A-Sample K). The error bar to each data point is the standard deviation of the Raman shift as the fitting parameter in our curve fitting program. Though the observed Raman modes are broad, the shift in this mode with the increase in the compactness of the sample could be seen even by the naked eye (marked by the.dashed line Fig. 4). From Fig. 7, the value of the slope $\left(\frac{\Delta v}{\Delta P}\right)$ is estimated to be $5.9 \times 10^{-9} \mathrm{~cm}^{-1} \mathrm{~Pa}^{-1}$. The calculated value of the compressibility $\kappa$ using the above expression is $(8.6 \pm 1) \times 10^{-12} \mathrm{~Pa}^{-1}$, which is comparable to the value $5 \times 10^{-12} \mathrm{~Pa}^{-1}$ for undoped $T-\mathrm{ZrO}_{2}$, as reported by Bouvier et al. [17]. To investigate the reversibility of the phase transition, we powdered the compacted pellet of the Sample K again and recorded its Raman spectrum. We name this sample as Sample $K^{\prime}$. The spectral profile carried the signatures of the irreversible nature of the phase transformation process (see the panels in the top in Fig. 4 for Sample $K^{\prime}$ ).

\section{Discussion}

The underlying mechanism for the observed compactioninduced phase transformation, as presented above, is expected to be different from what we find in most of the reports in the literature for $\mathrm{Y}_{2} \mathrm{O}_{3}$ and $\mathrm{CaO}$ doped systems under uniaxial or hydrostatic strain. Moreover, the compacting pressure applied on the pristine powder Sample A is an order of magnitude smaller than the same used to observe phase transition in the above mentioned doped compounds. To investigate the microscopic origin of the observed phase transformation in $\mathrm{ZnO}$ doped $\mathrm{ZrO}_{2}$ under nominal pressure, we studied the energetically relaxed crystal structure of the supercell of $\mathrm{ZnO}$ doped $\mathrm{ZrO}_{2}$ using DFT calculations. It is to be recalled that for the powder sample, $T$ - and $C$ - phases exist nearly in the ratio 1:1. From XRD and Raman scattering measurements (Figs. 3 and 5), we have observed an increase in the $M$-phase along with a decrease in the $T$-phase with the applied pressure. XRD patterns also revealed that the $C$-phase fraction remains nearly unchanged under compaction. Thus, to probe the effect of applied pressure on the crystal structure, we begin with the $T$-phase component in the crystal structure of $\mathrm{ZnO}$ doped $\mathrm{ZrO}_{2}$ to construct the supercell in our DFT calculations.

For comparison, we first simulate the optimized structures of undoped $\mathrm{ZrO}_{2}$ in the $M$ - and $T$-phase. The lattice constants of the pure $M$-phase (space group $P 2_{1} / c$ ) and $T$-phase (space group $\mathrm{P}_{2} / \mathrm{nmc}$ ), are taken from Ref. [26]. Fig. 8(a) and (b) show the unit cell of the undoped $M$-phase and the $T$-phase of $\mathrm{ZrO}_{2}$, respectively, as obtained from DFT calculations. The coordination numbers of $\mathrm{Zr}$ for $M-\mathrm{ZrO}_{2}$ and $\mathrm{T}-\mathrm{ZrO}_{2}$ are seven and eight, respectively [31]. To study the doped system, we first construct the supercell of $2 \times 2 \times 2$ unit cells of the $\mathrm{T}-\mathrm{ZrO}_{2}$ (with space group $\mathrm{P}_{2} / \mathrm{nmc}$ ) containing 48 atoms. For $\mathrm{ZnO}$ doped $\mathrm{ZrO}_{2}$, one $\mathrm{Zr}^{+4}$ ion is replaced by one $\mathrm{Zn}^{+2}$ ion. As there are $16 \mathrm{Zr}^{+4}$ ions in the supercell, on the replacement of a $\mathrm{Zr}^{+4}$ ion by a $\mathrm{Zn}^{+2}$ ion, we get $6.25 \% \mathrm{Zn}$ doped $\mathrm{ZrO}_{2}$. It is to be noted that this doping concentration of $\mathrm{Zn}$ is very close to the same 


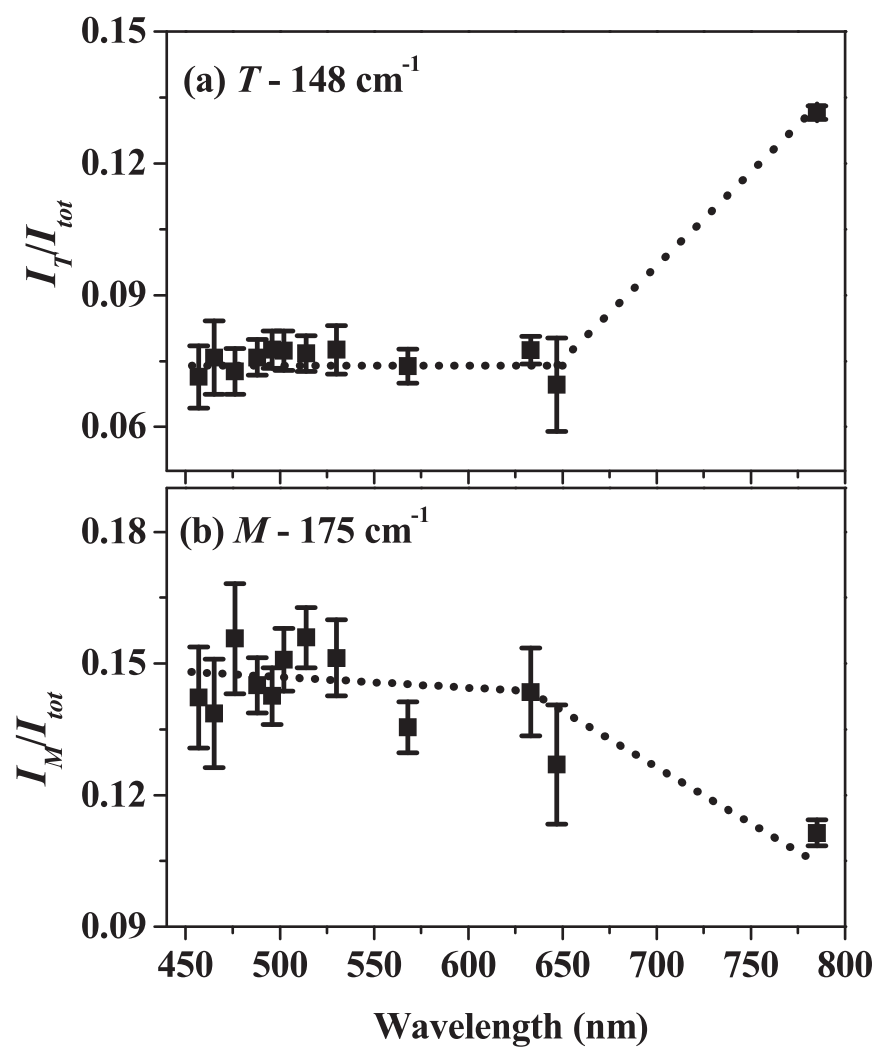

Fig. 6. The variation of the (a) $T$-fraction $\left(I_{T} / I_{\text {tot }}\right)$ by considering the intensity of the Raman mode at $148 \mathrm{~cm}^{-1}$ and (b) $M$-fraction $\left(I_{M} / I_{\text {tot }}\right)$ by considering the intensity of the Raman mode at $175 \mathrm{~cm}^{-1}$ as a function of incident wavelength for the Sample $\mathrm{K}$ (compacted at highest pressure $0.95 \mathrm{GPa}$ ). $I_{\text {tot }}$ is the net intensity, including the same of all modes. The error bar to each data point is obtained by using standard deviations of the intensities, as obtained from the curve fitting and following the propagation of error methodology. The dotted lines are guides to eyes.

that has been used experimentally ( $8 \%$ ) and involves computationally inexpensive simulation. An $\mathrm{O}$ vacancy around the $\mathrm{Zn}$ atom maintains charge neutrality. To obtain the initial atomic positions in the supercell structure, we have used the experimental lattice constants of the $T$-phase as obtained from the Rietveld refinement of the XRD pattern of the Sample A (refer to Table 1). Fig. 8(c) presents the DFT-relaxed crystal structure of $\mathrm{ZnO}$ doped $\mathrm{ZrO}_{2}$ of the $T$-phase. The doped $\mathrm{Zn}$ atom in the supercell is shown by a blue ball. The vacancy, introduced due to $\mathrm{Zn}^{2+}$ doping, is shown in the figure as a black ball and stick.

Table 3 lists $\mathrm{Zr}-\mathrm{O}$ bond lengths in the unit cells of undoped $M$ $\mathrm{ZrO}_{2}$ and $T-\mathrm{ZrO}_{2}$. For undoped $M$-phase, there are seven different $\mathrm{Zr}-\mathrm{O}$ bond lengths, whereas, for undoped $\mathrm{T}-\mathrm{ZrO}_{2}$, the crystal structure is relatively symmetric with two different sets of $\mathrm{Zr}-\mathrm{O}$ bond lengths. The simulated optimized structure of $\mathrm{Zn}^{2+}$ doped $\mathrm{ZrO}_{2}$ in Fig. 8(c) is expected to reveal the microscopic details of the crystal structure of the $T$-phase component of our doped system before compaction (mimic the powder Sample A). As the phase transformation involves changes in $\mathrm{Zr}-\mathrm{O}$ bond lengths, to investigate the local environment of the doped compound, we have compared various $\mathrm{Zr}-\mathrm{O}$ bond lengths in the undoped unit cell and doped supercell structures. A zoomed view of Fig. 8(c) is shown in Fig. 8(d). We find that in the supercell structure of the doped system, the nearest and the next nearest $\mathrm{Zr}$ atoms to $\mathrm{Zn}$ are in different environments, shown by the blue and the magenta polyhedra, respectively. We name these inequivalent $\mathrm{Zr}$ atoms as $\mathrm{Zr}$ 1 (in blue polyhedron) and $\mathrm{Zr} 2$ (in magenta polyhedron). The various bond lengths involving $\mathrm{Zr} 1$ and $\mathrm{Zr} 2$ with $\mathrm{O}$ atoms are also available in

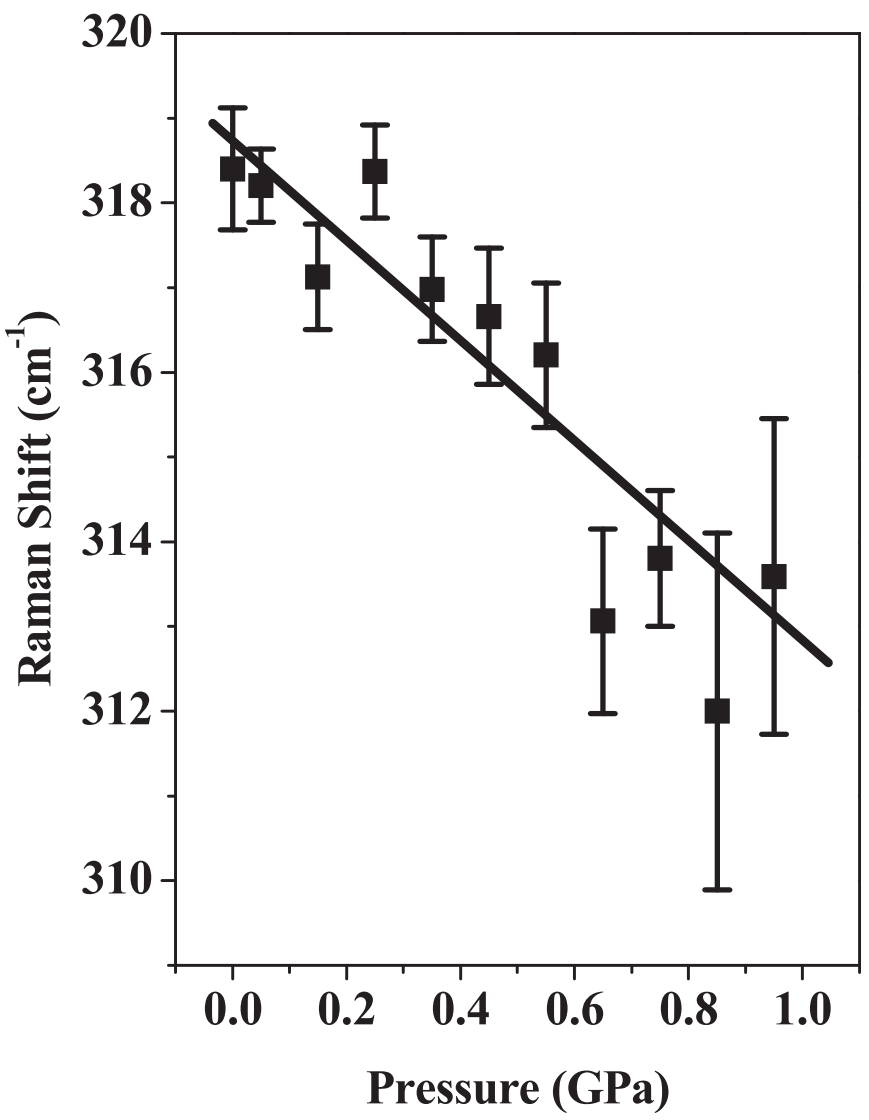

Fig. 7. The variation of the peak position of the $B_{1 g}$ mode $\left(318 \mathrm{~cm}^{-1}\right)$ as a function of applied pressure (Sample A-Sample K). The error bar to each data point is the standard deviation of the Raman shift (as a parameter) in the fitting procedure. The solid line is a fit to the data points using linear regression.

Table 3. To investigate the pressure-induced structural changes, we studied the optimized structure of the supercell under the highest pressure of $\sim 1 \mathrm{GPa}$, used in our experiments. The lattice constants of the supercell with and without applied pressure differed only by $\sim 0.6 \%$ (see Fig. 3(b)). This change is too small to exhibit any structural phase transition. $\mathrm{Zr} 1-\mathrm{O}$ and $\mathrm{Zr} 2-\mathrm{O}$ bond lengths in the compressed supercell of the doped compound are estimated to be very similar to the listed values in Table 3 for the same in the uncompressed supercell. Furthermore, since the ionic radius of $\mathrm{Zn}^{2+}$ ion ( $60 \mathrm{pm}$ [5]) is much smaller than that of $\mathrm{Zr}^{4+}$ ion (84 pm), the $\mathrm{Zn}$ substitution is expected to render a negative pressure. Thus, it is unusual to observe $T \rightarrow M$ phase transition under compacting pressure as the calculated volumes of the unit cell of $M$-phase $\left(138.9 \AA^{3}\right)$ is much larger than that of $T-\mathrm{ZrO}_{2}\left(66.9 \AA^{3}\right)$.

The laws of thermodynamics govern pressure-induced structural phase transition. The lattice parameters are distinctly different in different phases. Structural disorder determines the entropy and enthalpy of the states in a complex way and allows or inhibits the phase transition. To understand the experimentally observed pressure-induced phase transition, as described in the previous section, we look into the structural environment, especially the bonding environment of $\mathrm{Zr} 1$ and $\mathrm{Zr} 2$ atoms in the supercell of the doped system shown in Fig. 8(c) and (d). We find that the $\mathrm{Zr} 1$ atom has coordination number 7 (same as $\mathrm{Zr}$ in undoped $\mathrm{M}-\mathrm{ZrO}_{2}$ ). Though the $\mathrm{Zr} 1-\mathrm{O}$ bond lengths are very different from those of undoped $M$ - and $T$-phase $\mathrm{ZrO}_{2}$, the average $\mathrm{Zr} 1-\mathrm{O}$ bond length $(2.162 \AA)$ is very close to the same of the undoped $M-\mathrm{ZrO}_{2}$ (2.163 $\AA$ ). $\mathrm{Zr} 2$ atom, next nearest to the doping site, has the coordination number 8 (same as $\mathrm{Zr}$ in undoped $T-\mathrm{ZrO}_{2}$ ) 
(a)

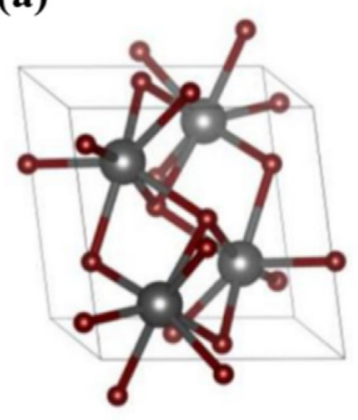

(b)

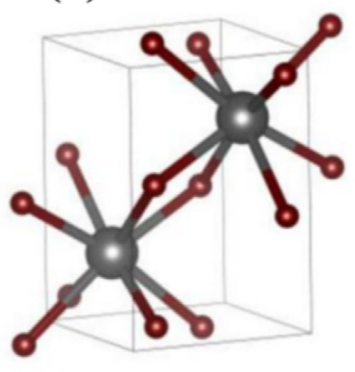

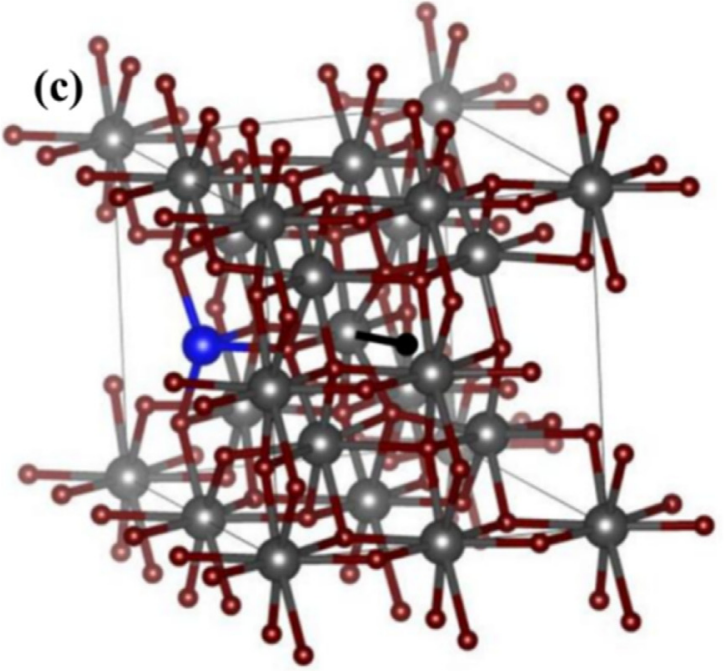

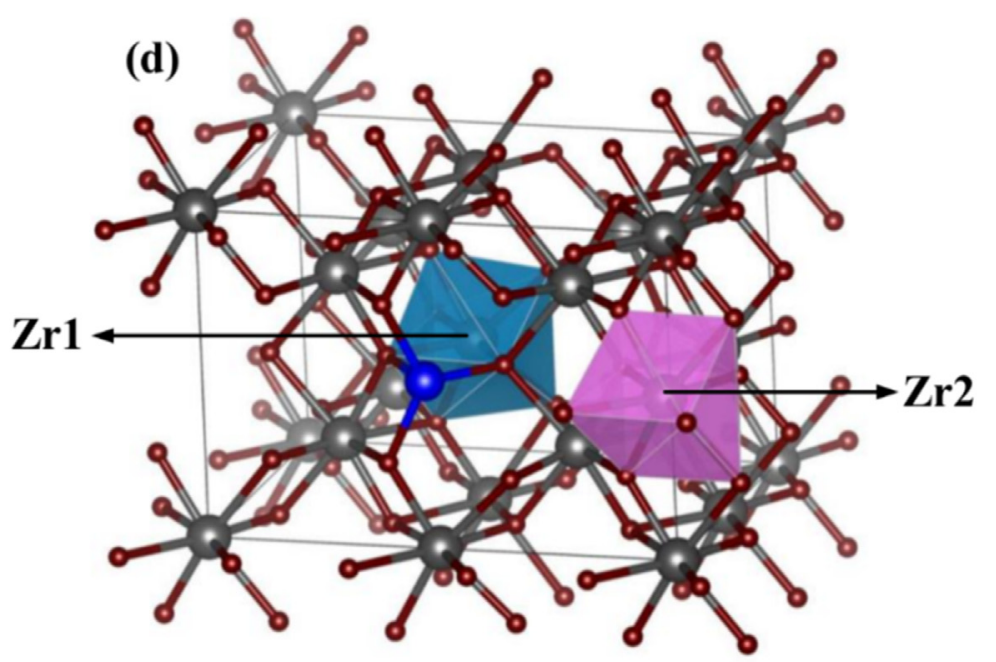

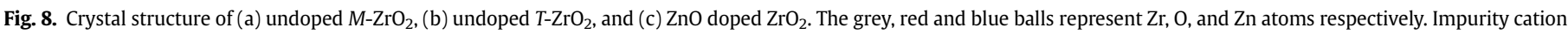

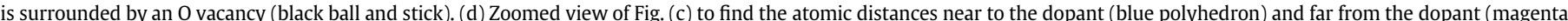

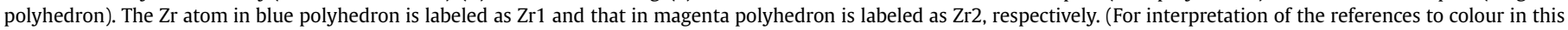
figure legend, the reader is referred to the Web version of this article.)

Table 3

$\mathrm{Zr}-\mathrm{O}$ bond lengths of undoped $M-, T-\mathrm{ZrO}_{2}$, and $\mathrm{ZnO}$ doped $\mathrm{ZrO}_{2}$.

\begin{tabular}{|c|c|c|c|}
\hline \multicolumn{4}{|c|}{$\mathrm{Zr}-\mathrm{O}$ bond distance in $\AA$} \\
\hline$M-\mathrm{ZrO}_{2}$ & $T-\mathrm{ZrO}_{2}$ Undoped & Zr1 (nearest to the doping site: in blue polyhedron) & $\mathrm{Zr} 2$ (next nearest to the doping site: in magenta polyhedron) \\
\hline \multicolumn{4}{|r|}{ 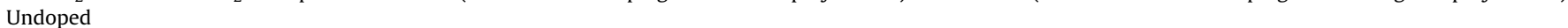 } \\
\hline 2.062 & 2.082 & 2.068 & 2.133 \\
\hline 2.065 & 2.082 & 2.109 & 2.133 \\
\hline 2.150 & 2.082 & 2.127 & 2.211 \\
\hline 2.187 & 2.082 & 2.127 & 2.216 \\
\hline 2.189 & 2.344 & 2.225 & 2.243 \\
\hline 2.240 & 2.344 & 2.225 & 2.265 \\
\hline \multirow[t]{2}{*}{2.243} & 2.344 & 2.254 & 2.265 \\
\hline & 2.344 & & 2.319 \\
\hline
\end{tabular}

and the individual bond lengths or the average bond length are very different from the values of the same in undoped $T$-phase, listed in Table 3. From above observations, we believe that the local structure around $\mathrm{Zr} 1$ atom is more susceptible to form the localized $M$-phase under an external perturbation (small pressure) in the real system at room temperature (DFT calculations are carried out for $\mathrm{T}=0$ ), while the $\mathrm{Zr} 2$ atom, next nearest to the doping site, gives rise to a low pressure-induced distorted $T$-phase( $T^{\prime}$-phase). However, more detail calculations are needed to comment conclusively on it.
It is noteworthy that the muffin-tin radii of $\mathrm{Zn}$ and $\mathrm{Zr}$ are comparable, 1.95 and 2.0, respectively, in the constructed doped supercell. Thus, it is reasonable to believe that though the ionic radius of tetrahedrally coordinated $\mathrm{Zn}^{2+}$ is much smaller than that of $\mathrm{Zr}^{4+}$ ( $60 \mathrm{pm}$ for $\mathrm{Zn}^{2+}$ in comparison to $84 \mathrm{pm}$ for $\mathrm{Zr}^{4+}$ ), the dopant can have a complex influence on the structural rearrangement of the host matrix.

As discussed earlier, a variety of pressure-induced structural responses are reported in the literature for doped zirconia. In all 
these studies, although the phase transitions in the zirconia can be observed as the final effect, it remains customary to understand the mechanism by which the $\mathrm{ZrO}_{2}$ matrix would accommodate the 'cause' factors (e.g., pressure, temperature, etc.) to induce or restrict a phase transition. We have shown the importance of the local structural environment, induced by doped metal cation, in understanding the phase integrity of the system. The appearance of the $M$-phase (by such low pressure) in $\mathrm{ZrO}_{2}$ is detrimental for any engineering and medical applications [32]. The strength of zirconia declines sharply with an increase in the $M$-phase quotient. We believe that the understanding of phase transformation at a pressure ( 1 GPa) has significant technological relevance as it matches with the pressure ranges for the shaping of the ceramics.

\section{Conclusion}

We have discussed the possible dynamics of structural phase evolution in $\mathrm{ZnO}$-doped $\mathrm{ZrO}_{2}$ under low scale ( $<1 \mathrm{GPa}$ ) compacting pressure, exploiting XRD, Raman scattering measurements, and DFT calculations. It is to be noted that the compacting pressure used in the present work is lower than the same reported in the literature for observing phase transition in doped and undoped $\mathrm{ZrO}_{2}$. The Rietveld refinements of XRD patterns reveal the partial transformation of the $T$-host matrix to $M$-phase and distorted $T^{\prime}$-phase. Raman spectral data confirm the evolution of the $M$-phase with the increase in the mechanical pressure and, in addition, indicate that the low symmetry phase forms only near-surface/subsurface region of the compacted block. The optimized supercell structure of the doped system, as obtained from the DFT calculations, reveals the importance of the local bonding environment around the dopant atom to explain the origin of the new phases, both $M$-and $T^{\prime}-$, in a real system, under external perturbation.

\section{Declaration of competing interest}

The authors declare that they have no known competing financial interests or personal relationships that could have appeared to influence the work reported in this paper.

\section{Acknowledgement}

SM thanks DST for financial support under WOS-A scheme (Ref. No.: SR/WOS-A/PM-38/2017(G)). SM and AR thank Dr. A. Singha, Bose Institute of Basic Sciences, Kolkata, for Raman measurements using $632 \mathrm{~nm}$ and $785 \mathrm{~nm}$ laser wavelengths.

\section{Appendix A. Supplementary data}

Supplementary data related to this article can be found at https://doi.org/10.1016/j.jallcom.2020.155927.

\section{References}

[1] S. Starschich, U. Bottger, Doped $\mathrm{ZrO}_{2}$ for future lead free piezoelectric devices, J. Appl. Phys. 123 (1-6) (2018), 044101.

[2] J. Chevalier, L. Gremillard, S. Deville, Low-temperature degradation of zirconia and implications for biomedical implants, Annu. Rev. Mater. Res. 37 (2007) $1-32$.

[3] M. Pesic, S. Knebel, M. Geyer, S. Schmelzer, U. Böttger, N. Kolomiiets, V.V. Afanasev, K. Cho, C. Jung, J. Chang, H. Lim, T. Mikolajick, U. Schroeder, Low leakage $\mathrm{ZrO}_{2}$ based capacitors for sub $20 \mathrm{~nm}$ dynamic random access memory technology nodes, J. Appl. Phys. 119 (1-5) (2016), 064101.

[4] S. Shibayama, T. Nishimura, S. Migita, A. Toriumi, Thermodynamic control of ferroelectric-phase formation in $\mathrm{Hf}_{x} \mathrm{Zr}_{1-x} \mathrm{O}_{2}$ and $\mathrm{ZrO}_{2}$, J. Appl. Phys. $124(1-7)$ (2018) 184101.
[5] R.D. Shannon, Revised effective ionic radii and systematic studies of interatomic distances in halides and chalcogenides, Acta Crystallogr. A 32 (1976) $751-767$.

[6] C.W. Li, H.L. Smith, T. Lan, J.L. Niedziela, J.A. Munoz, J.B. Keith, L. Mauger, D.L. Abernathy, B. Fultz, Phonon anharmonicity of monoclinic zirconia and yttrium-stabilized zirconia, Phys. Rev. B 91 (1-8) (2015) 144302.

[7] M. Ohta, J.K. Wigmore, K. Nobugai, T. Miyasato, Influence of dopant ion on localized relaxation of an oxygen vacancy in stabilized zirconia, Phys. Rev. B $65(1-8)(2002) 174108$.

[8] A. Sobolev, A. Musin, G. Whyman, K. Borodianskiy, O. Krichevski, A. Kalashnikov, M. Zinigrad, Stabilization of cubic phase in scandium-doped zirconia nanocrystals synthesized with sol-gel method, J. Am. Ceram. Soc. 102 (2019) 3236-3243.

[9] B. Alzyab, C.H. Perry, R.P. Ingel, High-pressure phase transitions in zirconia and yttria-doped zirconia, J. Am. Ceram. Soc. 70 (1987) 760-765.

[10] J. Cai, Y.S. Raptis, E. Anastassakis, Stabilized cubic zirconia: a Raman study under uniaxial stress, Appl. Phys. Lett. 62 (1993) 2781-2783.

[11] J. Wang, G. Li, Z. Li, C. Tang, Z. Feng, H. An, H. Liu, T. Liu, C. Li, A highly selective and stable $\mathrm{ZnO}-\mathrm{ZrO}_{2}$ solid solution catalyst for $\mathrm{CO}_{2}$ hydrogenation to methanol, Sci. Adv. 3 (1-10) (2017), e1701290.

[12] M.M. Ibrahim, Photocatalytic activity of nanostructured $\mathrm{ZnO}-\mathrm{ZrO}_{2}$ binary oxide using fluorometric method, Spectrochim. Acta Part A: Mol. Biomol. Spectrosc. 145 (2015) 487-492.

[13] E.D. Sherly, J.J. Vijaya, N.C.S. Selvam, L.J. Kennedy, Microwave assisted combustion synthesis of coupled $\mathrm{ZnO}-\mathrm{ZrO}_{2}$ nanoparticles and their role in the photocatalytic degradation of 2,4-dichlorophenol, Ceram. Int. 40 (2014) 5681-5691.

[14] A.O. de Souza, F.F. Ivashita, V. Biondo, A. Paesano Jr., D.H. Mosca, Structural and magnetic properties of iron doped $\mathrm{ZrO}_{2}$, J. Alloys Compd. 680 (2016) $701-710$.

[15] P. Bouvier, G. Lucazeau, Raman spectra and vibrational analysis of nanometric tetragonal zirconia under high pressure, J. Phys. Chem. Solid. 61 (2000) 569-578.

[16] W. Wang, Z. Liang, X. Han, J. Chen, C. Xue, H. Zhao, Mechanical and thermodynamic properties of $\mathrm{ZrO}_{2}$ under high-pressure phase transition: a firstprinciples study, J. Alloys Compd. 622 (2015) 504-512.

[17] P. Bouvier, E. Djurado, G. Lucazeau, T. Le Bihan, High-pressure structural evolution of undoped tetragonal nanocrystalline zirconia, Phys. Rev. B 62 (2000) 8731-8737.

[18] V. Milman, A. Perlov, K. Refson, S.J. Clark, J. Gavartin, B. Winkler, Structural, electronic and vibrational properties of tetragonal zirconia under pressure: a density functional theory study, J. Phys. Condens. Matter 21 (1-12) (2009) 485404.

[19] C.H. Perry, F. Lu, D.W. Liu, B. Alzyab, Phonons and phase transitions in zirconia, J. Raman Spectrosc. 21 (1990) 577-584.

[20] H. Öztürk, M. Durandurdu, High-pressure phases of $\mathrm{ZrO}_{2}$ : an ab initio constant-pressure study, Phys. Rev. B 79 (1-6) (2009) 134111

[21] D. Majumdar, D. Chatterjee, X-ray photoelectron spectroscopic studies on yttria, zirconia and yttria-stabilized zirconia, J. Appl. Phys. 70 (1991) 988-992.

[22] K. Kumar, T. Jaroń, A. Chowdhury, On the peculiarities of phase developments involving $\mathrm{Zn}^{2+}$-doped $\mathrm{ZrO}_{2}$ system, Scripta Mater. 138 (2017) 71-74.

[23] J. Rodriguez-Carvajal, Fullprof: A Program for Rietveld Refinement and Pattern Matching Analysis," Abstract of the Satellite Meeting on Powder Diffraction of the XV Congress of the IUCr, France, Toulouse, 1990, p. 127.

[24] P. Blaha, K. Schwarz, G. Madsen, , et al.K. Wien2, in: K. Schwarz (Ed.), An Augmented Plane Wave + Local Orbital Program for Calculating Crystal Properties, Technical University, Vienna, Austria, 2001.

[25] Z. Wu, R.E. Cohen, More accurate generalized gradient approximation for solids, Phys. Rev. B 73 (1-6) (2006) 235116.

[26] H. Tomaszewski, K. Godwod, Influence of oxygen partial pressure on the metastability of undoped zirconia dispersed in alumina matrix, J. Eur. Ceram. Soc. 15 (1995) 17-23.

[27] C.J. Howard, R.J. Hill, B.E. Reichert, Structures of $\mathrm{ZrO}_{2}$ polymorphs at room temperature by high-resolution neutron powder diffraction, Acta Crystallogr. B44 (1988) 116-120.

[28] B. Kim, J. Hahn, K. Han, Quantitative phase analysis in tetragonal-rich tetragonal/monoclinic two phase zirconia by Raman spectroscopy, J. Mater. Sci. Lett. 16 (1997) 669-671.

[29] P.P. Kyaw, P. Pongprueksa, W. Anuchitolarn, K. Sirinukunwatta, K. Suputtamongkol, Ageing assessment of zirconia implant prostheses by three different quantitative assessment techniques, J Adv Prosthodont 11 (2019) 253-261.

[30] V. Presser, M. Keuper, C. Berthold, K.G. Nickel, Experimental determination of the Raman sampling depth in zirconia ceramics, Appl. Spectrosc. 63 (2009) $1288-1292$.

[31] H. Ikeno, M. Krause, T. Hoche, C. Patzig, Y. Hu, A. Gawronski, I. Tanaka, C. Russel, Variation of $\mathrm{Zr}_{\mathrm{r}} \mathrm{L}_{2,3}$ XANES in tetravalent zirconium oxides, J. Phys, Condens. Matter 25 (1-8) (2013) 165505.

[32] A. Samran, A. Al-Ammari, S. El Bahra, E. Halboub, S. Wille, M. Kern, Bond strength durability of self-adhesive resin cements to zirconia ceramic: an in vitro study, J. Prosthet. Dent 121 (2019) 477-484. 